Conclusions: Despite treatment, patients with pSS still experience a high disease burden. Here we have provided novel insights into the higher treatment cost and increased healthcare utilisation burden of pSS compared with the sicca-free cohort, in particular for patients with extra-glandular disease manifestations. Acknowledgements: Study funded by GSK. Jennie Frain, PhD, Fishawack Indicia Ltd, UK, provided editorial assistance funded by GSK

Disclosure of Interest: S. Perera Shareholder of: GSK, Employee of: GSK, L. Ma Shareholder of: GSK, Employee of: GSK, R. Punwaney Shareholder of: GSK, Employee of: GSK, S. Ramachandran Shareholder of: GSK, Employee of: GSK DOI: 10.1136/annrheumdis-2017-eular.2352

\section{FRI0764-HPR MORTALITY IN PATIENTS WITH RHEUMATOID ARTHRITIS AND END STAGE RENAL DISEASE}

S. Paudyal ${ }^{1}$, J. Waller ${ }^{2}$, A. Oliver ${ }^{3}$, B. Le ${ }^{3}$, N. Zleik ${ }^{3}$, N.S. Nahman ${ }^{3,4}$, L. Carbone ${ }^{3,4}{ }^{1}$ Internal Medicine, School of Medicine, University of South Carolina, Columbia; ${ }^{2}$ Biostatistics; ${ }^{3}$ Medical College of Georgia, Augusta University; ${ }^{4}$ Charlie Norwood Veterans Affairs Medical Center, Augusta, United States

Background: Cardiovascular related mortality is higher in patients with rheumatoid arthritis (RA) compared to the general population, and accounts for more than half of all deaths in end stage renal disease (ESRD). The prevalence of ESRD is increasing and there are an increasing number of older patients with RA. Our recent study demonstrated approximately $1 \%$ of patients with ESRD have RA. The implications of ESRD on RA relative to the burdens of cardiovascular diseases, cardiovascular and all-cause mortality are not known.

Objectives: To determine whether patients with RA who have ESRD are at increased risk for cardiovascular disease (CVD) events, cardiovascular mortality and all-cause mortality compared to the general population of patients with ESRD. Methods: Retrospective cohort study of adult patients (age 18 and older) with ESRD receiving renal replacement therapy (hemodialysis or peritoneal dialysis) in the United States Renal Data System (USRDS) who initiated dialysis between 2005 and 2008 followed for up to five years. Patients with an ICD-9 diagnostic code for RA on or before the start of dialysis and a $5 \%$ random sample of those without RA were included. Incident cardiovascular events, cardiovascular related mortality and all-cause mortality was determined in those with RA compared to those without RA.

Results: There were 2,824 subjects including 407 with $R A$ and 2,417 without RA included in the analyses. There was no significant difference in the total number of incident CVD events by RA status ( $n=311(76.4 \%$ RA) vs. $n=1936(80.1 \%$ without RA) ( $p=0.09)$. 76 patients with RA (18.7\%) died from a CVD related cause compared to 403 without $R A(16.7 \%),(p=0.32)$. Overall mortality was significantly higher in those with RA ( $n=226(55.5 \%)$ vs. $n=970(40.1 \%)(p<0.01)$. Compared to those without RA, those with RA had a significantly shorter mean time in months from start of dialysis to any incident CVD event (17.5 (12.4)) vs. 21.2 (14.1) $(p<0.01)$, CVD death, (34.2 (12.5) vs.37.9 (12.6) $p=0.02)$, or all-cause mortality (33.1 (13.0) vs. $37.8(12.6)(p<0.01)$. In final adjusted models, RA was associated with an increased risk for both CVD related mortality (aHR=1.23 $(95 \%$ $\mathrm{Cl} 1.05-1.43)$ ) and all-cause mortality (aHR=1.22 (1.05 - 1.42) within five years. Risk factors for CVD and overall mortality included older age, a higher Charlson comorbidity index, tobacco use, needing assistance with ADLs and living in a nursing home. Black race and Hispanic ethnicity was associated with significantly less CVD and all cause-related mortality.

Conclusions: Physicians treating patients with RA and ESRD should be aware that these patients are at increased risk for cardiovascular related mortality and all-cause mortality compared with the general population of ESRD patients. Patients with ESRD and RA at higher risk for mortality can be identified by both demographic risk factors as well as overall health status.

Acknowledgements: Funding for this work was supported by the Translational Research Program (TRP) at the Medical College of Georgia and the Medical and Graduate Student Preceptorship award from the Rheumatology Research Foundation, Atlanta, Georgia.

Disclosure of Interest: None declared

DOI: 10.1136/annrheumdis-2017-eular.1152

\section{FRI0765-HPR PREVALANCE OF SARCOPENIA IN ELDERLY WITH OSTEOARTHRITIS OF LARGE JOINTS}

Y. Safonova ${ }^{1}$, V. Tsurko ${ }^{2} .{ }^{1}$ Northwestern State Medical University named after II Mechnikov, Department of gerontology, Geriatrics and Nursing, St. Petersburg;

${ }^{2}$ First Moscow State University named after IM Setchenov Department of Hematology and Geriatrics, Moscow, Russian Federation

Background: Lean muscle mass and strength decline starting approximately at 40 years of age to become $25 \%$ of body weight at $75-80$ years old [1]. Within the existing literature, sarcopenia is a highly prevalent condition in older people. The prevalence of sarcopenia increases considerably with age ranging from $5 \%$ to $13 \%$ in 60 to 70 years, from $11 \%$ to $50 \%$ for the population aged 80 years and older. In older persons, sarcopenia is related to falls and physical disability leading to reduced quality of life [2]. The prevalence of osteoarthritis increases with age so that 30 to $50 \%$ of adults over the age of 65 years suffer from this condition [3]. Age-related factor that contributes includes to the development of OA include a decline in muscle strength. People with lower extremity OA had a two to five times increased incidence of falls than age-matched healthy controls [4].

Objectives: Conduct analysis of condition of muscle strength and muscle functioning in older persons with osteoarthritis.

Methods: Prospective study of 159 patients aged $74 \pm 13,3$ years was held. Condition of sarcopenia was estimated by lean body mass (LBM) in accordance with criteria of sarcopenia EWGSOP. Muscle strength was estimated by a hand dynamometer and muscle functioning was estimated on the basis of SPPB tests. Amount of pain was estimated by VAS.

Results: Sarcopenia was revealed in $31,45 \%$ of older persons with ostearthritis. Cases of falls were observed in $28,30 \%(95 \% \mathrm{Cl} 21,5-36,0)$ in patients with osteoartritis with sarcopenia (average number of falls $-1,93$ ) and in $16,98 \%$ of patients without sarcopenia $(95 \% \mathrm{Cl} 11,5-23,7)$ (average number of falls 0,48 ). Level of pain in patients with osteoartritis with sarcopenia amounted 3,16 points, in patients without sarcopenia $-3,49$ points $(p>0,05)$. Muscle strength in patients with sarcopenia was $14,36 \mathrm{~kg}$, in patients without sarcopenia was significantly higher $-18,53 \mathrm{~kg}(p<0,05)$. Common point of SPPB tests in patients with sarcopenia was 6,9 , in patients without sarcopenia significantly higher $-7,85$ $(p<0,05)$

Conclusions: Patients with sarcopenia in the presence of osteoarthritis were observed to have significant decrease of muscle strength and muscle functioning, increase of frequency of falls which raises risk of repeated falls and their frequency, and consequently, deteriorates condition of musculoskeletal system in older persons.

References:

[1] Ferrucci L., Baroni M., Ranchelli A. et al. Interaction Between Bone and Muscle in Older Persons with Mobility Limitations. Curr Pharm Des. 2014; 20(19): 3178-3197.

[2] Morley JE. Sarcopenia: diagnosis and treatment. J Nutr Health Aging. 2008; 12:452-456.

[3] Felson DT. Risk factors for osteoarthritis: understanding joint vulnerability. Clinical orthopaedics and related research 2004;(427 Suppl):S16-21.

[4] Hoops ML, Rosenblatt NJ, Hurt CP, Crenshaw J, Grabiner MD. Does lower extremity osteoarthritis exacerbate risk factors for falls in older adults? Womens Health (Lond Engl). 2012;8(6):685-96.

Disclosure of Interest: None declared

DOI: 10.1136/annrheumdis-2017-eular.3097

\section{FRIDAY, 16 JUNE 2017 \\ HPR professional education, training and competencies}

\section{FRI0766-HPR DOWN'S ARTHROPATHY - CLINICAL AND RADIOLOGICAL FEATURES OF ARTHRITIS IN CHILDREN WITH TRISOMY 21}

\section{Foley, E.J. MacDermott, O.G. Killeen. OLCHC, Dublin, Ireland}

Background: Down's Arthropathy (DA) was first reported in the literature in 1984. Crude estimates suggest higher incidence and prevalence rates of DA compared with Juvenile Idiopathic Arthritis (JIA), (JIA prevalence 1/1000, estimated DA prevalence $8.7 / 1000$ ). Despite this fact, there remains a paucity of data on this condition. DA is rarely recognised at onset, \& remains under-diagnosed. As a direct consequence children with DA are presenting with significant joint damage and disability at diagnosis.

Objectives: Perform a musculoskeletal examination on children with Trisomy 21 (T21) aged $0-20$ years

Methods: Children with T21 were invited to attend a screening clinic. Screening involved completion of a health questionnaire \& a comprehensive musculoskeletal examination. DA cases detected were investigated \& managed as per normal clinical practice. Data on a convenience sample of 33 newly diagnosed children with JIA was collected to create a comparison group.

Results: 503 children with T21 have been screened for DA, 22 new cases have been diagnosed. All of these children had poor language skills or were non-verbal. Only $11 \%$ of the parents suspected that their child may have arthritis prior to attending our screening clinics, and this was only after reading our recruitment literature. In total, we now have 33 children attending our centre with DA (combining cases attending pre-dating the start date of the study). This suggests the prevalence of DA in Ireland is $18-21 / 1000$.

The majority of children presented with a polyarticular pattern of disease. No cases of uveitis have been observed to date. $88 \%$ of the DA cohort had small joint involvement of the hands, significantly higher than that observed in the JIA comparison group. Erosive changes were reported on X-Ray in $29.2 \%$ of the DA cohort (9.5\% in the JIA Cohort). Methotrexate-associated nausea was a significant barrier to treatment with this DMARD in DA. There was a significant delay in diagnosis of DA, 1.7 years $v 0.7$ years in the JIA cohort.

Conclusions: Children with T21 are at increased risk of developing arthritis. There is a lack of awareness of this risk among health care professionals \& the general public at large. This almost certainly contributes to poor recognition of the disease and a delay in diagnosis. The predominant pattern of disease is polyarticular small joint arthritis. Treatment with standard protocols used in JIA 
is complicated by drug-associated side effects in children with T21. However, a good response to treatment with steroid intra-articular joint injections has been observed. Our study has raised a number of questions. Future research to accurately define this disease \& identify best practice with regards to treatment would be invaluable. We advocate that all children with T21 should have annual musculoskeletal examination as part of their health surveillance programme.

Disclosure of Interest: None declared

DOI: 10.1136/annrheumdis-2017-eular.7020

\section{FRI0767-HPR PARTICIPATING IN A MUSCULOSKELETAL RANDOMISED CONTROLLED TRIAL: IDENTIFICATION OF EDUCATION TRAINING NEEDS BY OCCUPATIONAL THERAPISTS AND PHYSIOTHERAPISTS IN THE UK}

J. Adams $^{1,2}$, P. Barratt ${ }^{2}$, S. Bradley ${ }^{3}$, S. Barbosa-Bouças ${ }^{4}$, K. Hislop Lennie ${ }^{5}$, P. White ${ }^{2}$ on behalf of OTTER II Trial working group. ${ }^{1}$ Arthritis Research UK, Centre of Excellence Sport, Ex \& OA; ${ }^{2}$ Faculty of Health Sciences, University of Southampton, Southampton; ${ }^{3}$ Occupational Therapy, Poole General Hospital, Poole: ${ }^{4}$ Department of Psychology, Buckinghamshire New University, High Wycombe; ${ }^{5}$ Health Sciences, University of Southampton, Southampton, United Kingdom

Background: There is an association between clinical teams engaging with research and improvement in the delivery of health services ${ }^{1}$. Randomised controlled trials (RCTs) provide strong evidence to influence practice in musculoskeletal services. For occupational therapists (OTs) and physiotherapists (PTs) implementing RCTs is not yet commonplace. As part of a multi-centred clinical effectiveness and efficacy RCT of splints for thumb base osteoarthritis (OTTER II Trial) we established an education training programme to support clinical therapists deliver the trial across 15 UK hospitals.

Objectives: To evaluate the content of trial training to educate and support OTTER II Trial clinicians in undertaking clinical research roles.

Methods: Two trial training days were run in the North and South of England. Therapists provided details of their clinical trial experience. They were asked to identify one area in which they felt confident and one in which they were not confident in participating in a RCT. These perceived facilitators and barriers were summarised using descriptive statistics and content analysis.

Results: Thirty five clinicians (20 OTs, 15 PTs) attended a training day, 13 (37\%) had no previous experience with clinical trials; $19(54 \%)$ had been involved with at least one previous clinical trial. Clinicians considered they were already confident in; delivering the trial standardised assessment and treatment to patients $n=21(60 \%)$; trusting the OTTER II trial team and their own hospital research departments to support their research role $n=11(31 \%)$; understanding the trial protocol and what needed to be delivered $n=6(17 \%)$ and, being convinced that the trial asked a relevant question $3(9 \%)$. Areas in which clinicians considered they did not have confidence included; the logistics and time management of delivering a RCT in their own hospital $n=18(51 \%)$; the associated trial paperwork to be completed $n=8(23 \%)$; NHS computer access/wifi access for randomisation procedure $n=7(20 \%)$; recruiting participants to time and target $n=6(17 \%)$ and staff capacity to deliver a RCT in the NHS alongside clinical commitments $n=5(14 \%)$. Conclusions: Well documented trial protocols and support from a trusted research team and local hospital research departments were identified as key areas that help clinicians become confident to engage with a national clinical RCT. Clinicians are less confident about managing the practical logistics, staff time and trial paperwork involved in delivering a national RCT. Clinicians identify that they possess core clinical assessment and treatment skills that already equip them to recruit and treat patients as part of a national trial. The logistics of delivering a trial requires ongoing negotiation and support from clinical service managers and the clinical trial teams to ensure clinicians are supported to deliver the RCT to time and target.

References:

[1] Boaz et al 2015 BMJ Open 5:e009415 doi:10.1136/bmjopen-2015-009415 Acknowledgements: The OTTER II Trial is funded by Arthritis Research UK (Grant Ref number 21019).

Disclosure of Interest: None declared

DOI: 10.1136/annrheumdis-2017-eular.1095

\section{FRI0768-HPR MUSCLE WASTING IN OSTEOARTHRITIS MODEL INDUCED BY ANTERIOR CRUCIATE LIGAMENT TRANSECTION}

J.M.D.S. Silva, P.V.G. Alabarse, V.D.O.N. Teixeira, E.C. Freitas, F.H. de Oliveira, R.M.D.S. Chakr, R.M. Xavier. Faculdade de Medicina, UFRGS, Porto Alegre, Brazil

Background: Osteoarthritis $(\mathrm{OA})$ is a chronic joint disease characterized by progressive loss of articular cartilage and abnormal bone formation. Furthermore, there are changes in periarticular muscles, such as loss of muscle mass, strength and function. These features may contribute to functional impairment among patients.

Objectives: This study aimed to investigate the molecular pathways involved in muscle wasting in an animal model of OA induced by anterior cruciate ligament $(\mathrm{ACL})$ transection in rats.
Methods: Female Wistar rats were allocated into two groups: OA (submitted to the ACL transection; $n=9$ ) and SHAM (submitted to surgical procedures without ACL transection; $n=8$ ) [1]. Spontaneous exploratory locomotion, nociception and body weight of animals were evaluated weekly. Twelve weeks after the disease induction, animals were euthanized and the right knee joints were collected for further confirmation of the disease by histopathology, accordingly to OARSI histologic scoring system [2]. Gastrocnemius muscle from the right hind paw were dissected and weighed. Gastrocnemius was used for evaluation of muscle atrophy [3] and protein expression of myostatin, MuRF-1, MyoD and myogenin. Data were compared by Student's $t$ test or ANOVA followed by Tukey's test or ANOVA followed by Mann-Whitney's U-test. The results are expressed as mean values \pm standard deviation (SD) for symmetric variables and as medians with interquartile range for asymmetric variables. Significance was accepted at $P<0.05$.

Results: Histopathology of the right knee joints confirmed the development of the disease in animals from OA group. Gastrocnemius area of animals from OA group had a reduction of about $10 \%$ compared to animals from SHAM group. Protein expression of myostatin was increased in OA group, while myogenin expression was decreased. MuRF-1 and MyoD expression was similar in both OA and SHAM groups. Spontaneous exploratory locomotion, nociception, body weight and weight of gastrocnemius showed no difference between OA and SHAM groups. Conclusions: Gastrocnemius atrophy in $\mathrm{OA}$ induced by $\mathrm{ACL}$ transection involves increased protein expression of myostatin and decreased protein expression of myogenin. In this model, muscle wasting may be linked to myostatin-induced deficits in satellite-cell differentiation due to decreased expression of myogenin.

References:

[1] Elsaid KA, Machan JT, Waller K, Fleming BC, Jay GD. The Impact of Anterior Cruciate Ligament Injury on Lubricin Metabolism and the Effect of Inhibiting Tumor Necrosis Factor alpha on Chondroprotection in an Animal Model. Arthritis and Rheumatism. 2009;60(10):2997-3006.

[2] Gerwin N, Bendele AM, Glasson S, Carlson CS. The OARSI histopathology initiative - recommendations for histological assessments of osteoarthritis in the rat. Osteoarthritis and Cartilage. 2010;18:S24-S34

[3] Filippin LI, Teixeira VN, Viacava PR, Lora PS, Xavier LL, Xavier RM. Temporal development of muscle atrophy in murine model of arthritis is related to disease severity. Journal of Cachexia Sarcopenia and Muscle. 2013:4(3):231-8.

[4] de Oliveira Nunes Teixeira V, Filippin LI, Viacava PR, de Oliveira PG, Xavier RM. Muscle wasting in collagen-induced arthritis and disuse atrophy. Exp Biol Med (Maywood). 2013:238(12):1421-30.

Disclosure of Interest: None declared

DOI: 10.1136/annrheumdis-2017-eular.4856

\section{FRI0769-HPR EVALUATION OF THE EFFECTIVENESS OF DEEP WATER RUNNING FOR THE TREATMENT OF CHRONIC NONSPECIFIC LOW BACK PAIN}

J.S. Arakaki, F.M. Jennings, S.R. Toffolo, J.C. Tamashiro, J. Natour. Rheumatology, Universidade federal de São Paulo- UNIFESP, São Paulo, Brazil

Background: Low back pain (LBP) is one of the most common musculoskeletal conditions and can lead to disability. Aerobic fitness exercises have recently been suggested as important in the management of pain and physical disability of LBP patients, but there are still no studies that prove the best exercise modality for this condition. One proposed modality is the Deep Water Running (DWR) which are aquatic conditioning exercises that simulates normal running on soil.

Objectives: To evaluate the effectiveness of Deep Water Running in the treatment of chronic nonspecific low back pain.

Methods: It was a randomized controlled trial with a duration of 16 weeks with evaluations performed before the intervention and 8 and 16 weeks after the beginning of the training. The population was composed of 60 patients aged over 18 years with diagnosis of chronic nonspecific low back pain. The intervention group performed aerobic conditioning exercise (Deep Water Running) in a heated pool. The sessions had duration of 50 minutes and frequency of 3 times a week for 16 weeks and with $70 \%$ of the maximum heart rate, with $10 \mathrm{bpm}$ less for the difference in the behavior of $\mathrm{HR}$ in aquatic environment. The control group underwent aerobic conditioning by treadmill exercise for 50 minutes, 3 times a week for 16 weeks, and also with $70 \%$ of maximal heart rate. Evaluation instruments: Visual analog pain scale (EVA) in cm; Likert Scale of pain improvement and worsening according to the patient and according to the evaluator; Functional capacity through the Roland-Morris questionnaire and 6-minute walk test; SF-36 for general quality of life; And amount of antiinflammatories used during the study period. Evaluations were performed by an evaluator who was unaware of the patient allocation group.

Results: The two groups were homogeneous regarding most clinical demographic characteristics in the initial evaluation. The two groups showed statistically significant improvement in the variables Roland Morris, EVA for pain and Time up and go, but no statistically significant differences were found between groups. At the Likert scale, the 6-minute walk test and the amount of anti-inflammatories used during the study, no significant differences were found, the two groups remained unchanged. No adverse events were observed in either group during the exercise program

Conclusions: Deep water running aerobic exercise is as effective as treadmill walking in improving pain and functional capacity in patients with chronic nonspecific low back pain. 